\title{
KLRfome - Kernel Logistic Regression on Focal Mean Embeddings
}

\author{
Matthew D. Harris ${ }^{1}$
}

1 AECOM Technologies

DOI: $10.21105 /$ joss.00722

\section{Software}

- Review ¿

- Repository ca

- Archive e

Submitted: 14 April 2018

Published: 19 March 2019

\section{License}

Authors of papers retain copyright and release the work under a Creative Commons Attribution 4.0 International License (CC-BY).

\section{Summary}

KLRfome is a package for predicting a geographic area's relative sensitivity for the presence of archaeological sites. This is achieved by fitting, predicting, and visualizing a Kernel Logistic Regression model on mean feature embeddings. This regression algorithm and package are created to improve upon the current methods in archaeological predictive modeling. These improvements include 1) modeling rich descriptions of archaeological land forms by mitigating undesirable spatial correlation between samples, 2) explicitly modeling the similarity between archaeological sites as characterized by rich features, 3) the ability to define research specific similarity measures, and 4) focal window prediction that can be modified based on theory or management goals.

More specifically, this package seeks to solve the Distribution Regression problem for archaeological site location modeling; or any other data for that matter. The aim of Distribution Regression is to map a single scalar outcome (e.g. presence/absence; 0/1) to a distribution of features. This is opposed to typical regression where you have one observation mapping a single outcome to a single set of features/predictors. For example, an archaeological site is singularly defined as either present or absent, however the area within the sites boundary is not singularly defined by any one measurement. The area with an archaeology site is defined by an infinite distribution of measurements. Modeling this in traditional terms means either collapsing that distribution to a single measurement or pretending that a site is actually a series of adjacent, but independent measurements. The methods developed for this package take a different view instead by modeling the distribution of measurements from within a single site on a scale of similarity to the distribution of measurements on other sites and the environmental background in general. This method avoids collapsing measurements and promotes the assumption of independence from within a site to between sites. By doing so, this approach models a richer description of the landscape in a more intuitive sense of similarity.

To achieve this goal, the package fits a Kernel Logistic Regression (KLR) model onto a mean embedding similarity matrix and predicts as a roving focal function of varying window size. The name of the package is derived from this approach; Kernel Logistic Regression on FOcal Mean Embeddings (klrfome) pronounced clear foam. This work is based on Szabó's work on feature mean embeddings for distribution regression (Szabó, Sriperumbudur, Póczos, \& Gretton, 2016, Szabó, Gretton, Póczos, \& Sriperumbudur (2015)) and the Kernel Logistic Regression algorithm in (Zhu \& Hastie, 2005). A poster introducing the KLRfome package was presented on April 12, 2018 at the Society for American Archaeology annual meeting in Washington, D.C.

The $\mathrm{R}$ package to implement this KLRfome model contains functions formatting tabular data format_data() into the a specified list format including training and testing data splits, building a Gaussian (RBF) similarity kernel build_k(), fitting the klr model klr(), 
and predicting the fit model to a stack of raster inputs KLR_raster_predict(). The hyperparameters of this model are sigma to control the variance of the Gaussian kernel, lambda to control the regularization penalty of the regression model, and ngb to control the neighborhood dimensions for the focal prediction function. The also package contains functions to simulate data get_sim_data() and correlated trend rasters sim_trend (using the NLMR package (Sciaini, Fritsch, \& Simpkins, 2017)) that proxy archaeological site locations and environments so that the package can be tested on smaller and less sensitive data sets. Finally, with an appropriate parallel processing backend, such as doParallel, the KLR_raster_predict() function can be parameterized to split the prediction area into blocks (split, ppside, and parallel arguments) and process each block on a separate core in parallel. Each block will be collared by the focal window ngb size so that when merged back together there is no edge effect from predicting as blocks.

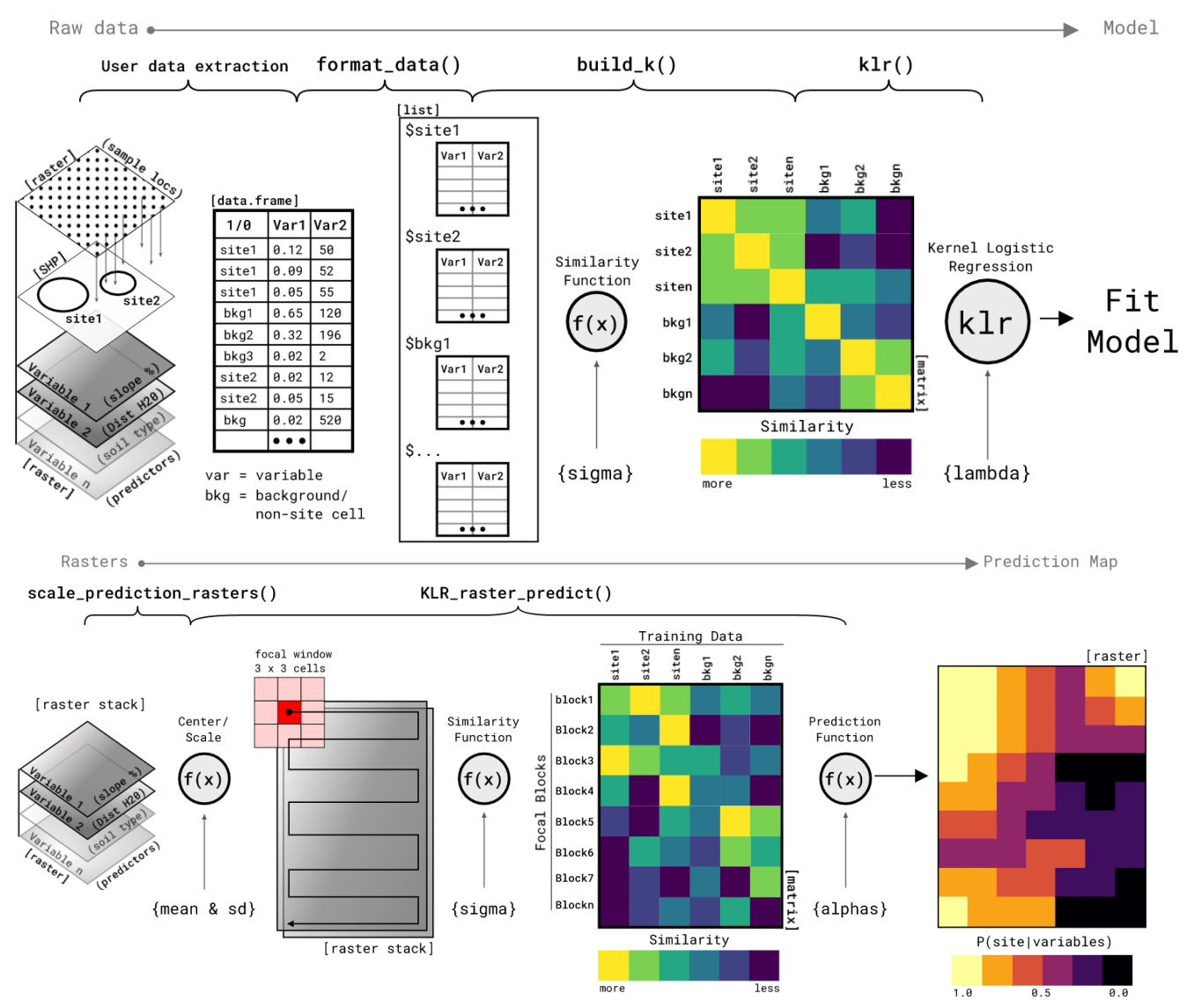

\section{References}

Sciaini, M., Fritsch, M., \& Simpkins, C. E. (2017). NLMR: Simulating neutral landscape models. Retrieved from https://CRAN.R-project.org/package=NLMR

Szabó, Z., Gretton, A., Póczos, B., \& Sriperumbudur, B. (2015). Two-stage sampled learning theory on distributions. In International conference on artificial intelligence and statistics (aistats) (pp. 948-957).

Szabó, Z., Sriperumbudur, B., Póczos, B., \& Gretton, A. (2016). Learning theory for distribution regression. Journal of Machine Learning Research, 17, 1-40.

Zhu, J., \& Hastie, T. (2005). Kernel logistic regression and the import-vector machine. Journal of Computational and Graphical Statistics, 14(1), 185-205. doi:10.1198/ 
$106186005 \times 25619$

Harris, (2019). KLRfome - Kernel Logistic Regression on Focal Mean Embeddings. Journal of Open Source Software, 4(35), 722. https: 3 //doi.org/10.21105/joss.00722 